\title{
Bystander hit by leakage current from S-ICD
}

\author{
Peran David ${ }^{1,2}$, Pekara Jaroslav ${ }^{1,3}$, Cmorej Patrik Christian $^{4,5}$ \\ ${ }^{1}$ Prague Emergency Medical Services, Czech Republic \\ ${ }^{2}$ Division of Public Health, 3rd Faculty of Medicine, Charles University in Prague, Czech Republic \\ ${ }^{3}$ Medical College, Prague, Czech Republic \\ ${ }^{4}$ Emergency Medical Services of the Usti nad Labem Region, Czech Republic \\ 5 Jan Evangelista Purkyne University, Faculty of Health Studies, Usti nad Labem, Czech Republic
}
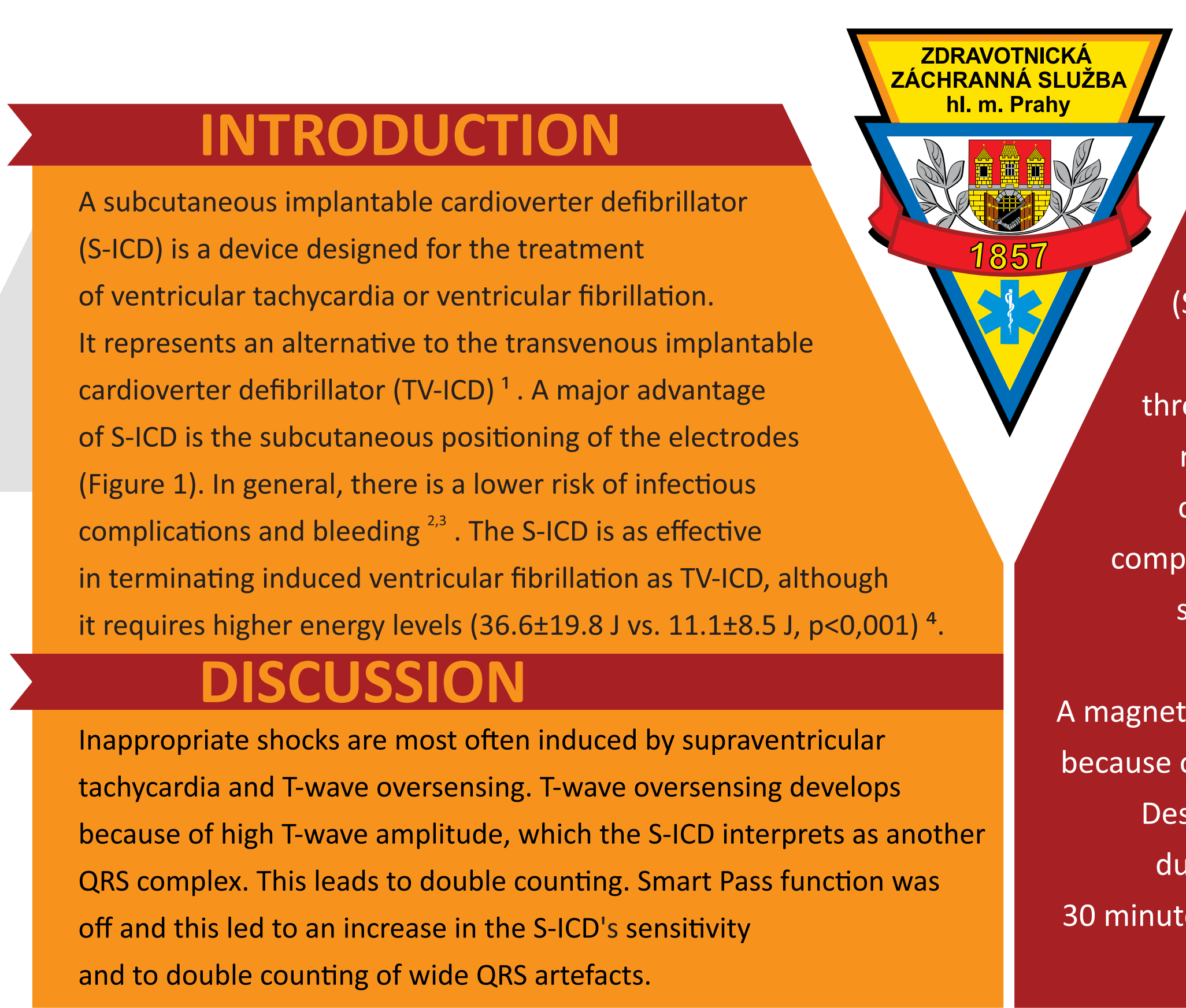

\section{CASE REPORT \\ We present the case of a 30 -year-old man with a subcutaneous implantable cardioverter defibrillator (S-ICD) who suffered sudden out-of-hospital cardiac arrest. \\ Despite rhythm on the EMS monitor showed asystole \\ throughout the period of resuscitation (Figure 2), the patient received inappropriate shocks due to oversensing of chest compressions (Figure 3) and a bystander performing chest compressions received a shock delivered by the S-ICD. This was \\ so unpleasant that the bystander refused to continue with} chest compressions.

A magnet - M50 (Biotronic Company) - was placed over the device because of repeated defibrillation shocks from S-ICD had occurred.

Despite these measures, further S-ICD shocks were delivered during the resuscitation. Resuscitation was terminated after 30 minutes, once all therapeutic options and all possible reversible causes of cardiac arrest had been exhausted.

\section{RECOMMENDATION}

1 Visible scars on the chest in a typical location should alert the paramedics to the presence of an S-ICD (Figure 4).

2 The S-ICD manufacturer (Boston Scientific) recommends using the 6860 magnet from Boston Scientific or the $\mathbf{4 5 2 0}$ magnet from Cameron Health. To inhibit shock delivery, the magnet must be placed above the upper or lower edge of the S-ICD generator. Successful inhibition of shock delivery by the magnet is signalised acoustically as beeping for 60 seconds. If no acoustic signal is detected following the magnet's placement above the upper or lower edge, then the magnet must be moved vertically and horizontally in the target zone as shown by the arrows (Figure 5).

3 The external electrodes should be placed as far away as possible from the S-ICD components. The authors of this case report favour the anterior-posterior positioning of external defibrillator electrodes.

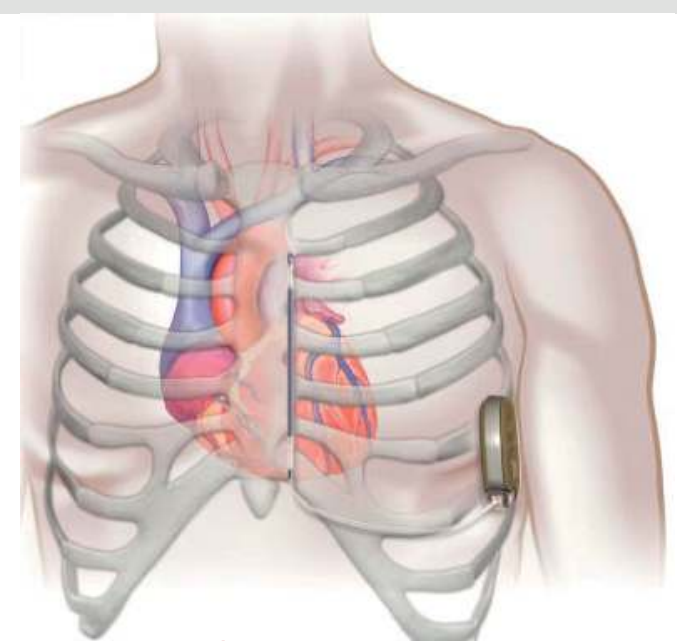

Figure 1

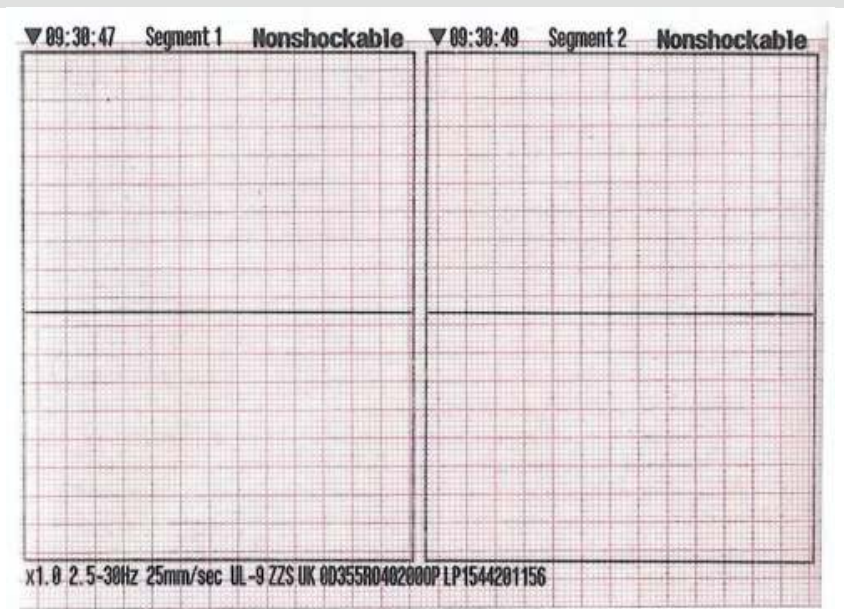

Figure 2

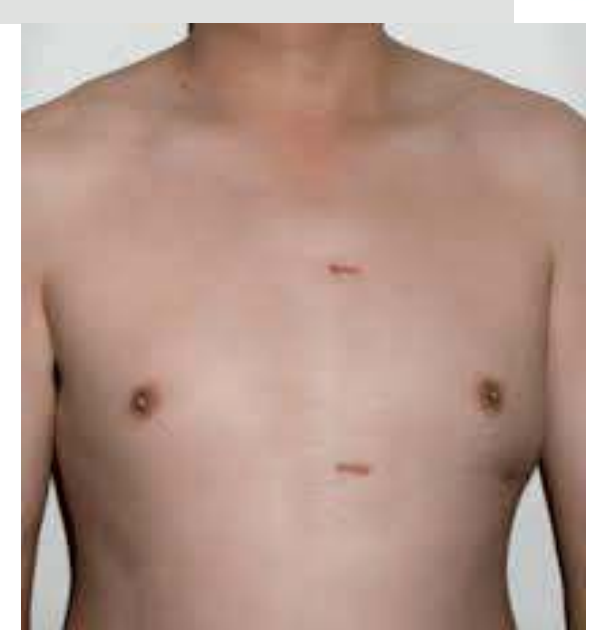

Figure 4

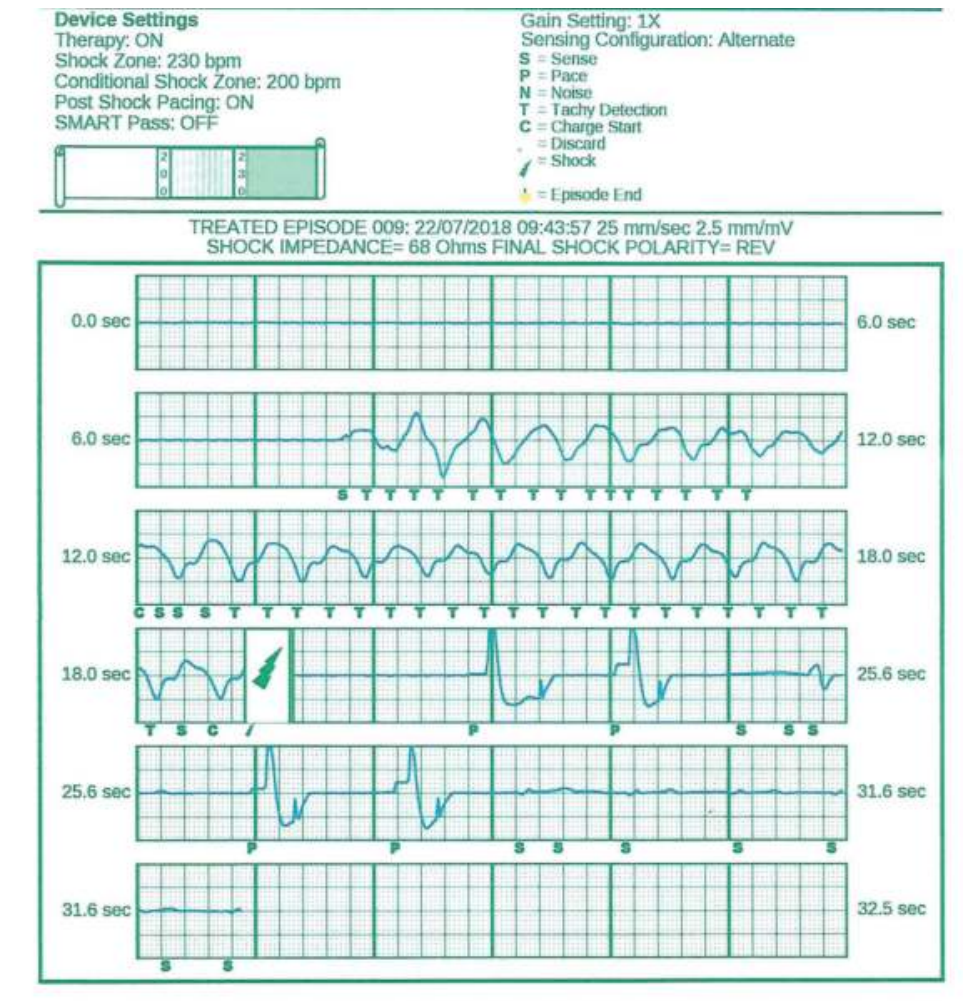

Figure 3

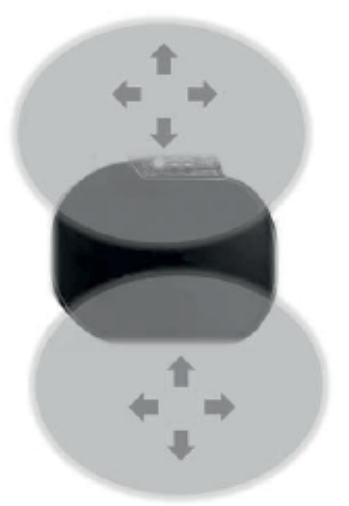

Figure 5

\section{CONCLUSION}

Out-of-hospital resuscitation of a patient with an implanted S-ICD places greater demands on the

knowledge, experience and skills of EMS paramedics and physicians. Inappropriate shocks and the specificities of magnet placement to inhibit shock delivery may decrease the quality of resuscitation.

\section{REFERENCES}

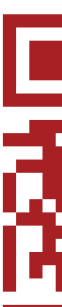

